\title{
Prader-Willi-like phenotypes: a systematic review of their chromosomal abnormalities
}

\author{
C.F. Rocha ${ }^{1}$ and C.L.A. Paiva ${ }^{1,2}$ \\ ${ }^{1}$ Programa de Pós-Graduação em Neurologia, \\ Hospital Universitário Gafrée e Guinle, Rio de Janeiro, RJ, Brasil \\ ${ }^{2}$ Programa de Pós-Graduação em Genética e Biologia Molecular, \\ Universidade Federal do Estado do Rio de Janeiro, Rio de Janeiro, RJ, Brasil \\ Corresponding author: C.L.A. Paiva \\ E-mail: clapaiva1@gmail.com
}

Genet. Mol. Res. 13 (1): 2290-2298 (2014)

Received January 15, 2014

Accepted February 27, 2014

Published March 31, 2014

DOI http://dx.doi.org/10.4238/2014.March.31.9

\begin{abstract}
Prader-Willi syndrome (PWS) is caused by the lack of expression of genes located on paternal chromosome 15q11-q13. This lack of gene expression may be due to a deletion in this chromosomal segment, to maternal uniparental disomy of chromosome 15 , or to a defect in the imprinting center on 15q11-q13. PWS is characterized by hypotonia during the neonatal stage and in childhood, accompanied by a delay in neuropsychomotor development. Overeating, obesity, and mental deficiency arise later on. The syndrome has a clinical overlap with other diseases, which makes it difficult to accurately diagnose. The purpose of this article is to review the Prader-Willi-like phenotype in the scientific literature from 2000 to 2013, i.e., to review the cases of PWS caused by chromosomal abnormalities different from those found on chromosome 15. A search was carried out using the "National Center for Biotechnology Information" (www.pubmed.com) and "Scientific Electronic Library Online (www.scielo.br) databases and
\end{abstract}


combinations of key words such as "Prader-Willi-like phenotype" and "Prader-Willi syndrome phenotype". Editorials, letters, reviews, and guidelines were excluded. Articles chosen contained descriptions of patients diagnosed with the PWS phenotype but who were negative for alterations on 15q11-q13. Our search found 643 articles about PWS, but only 14 of these matched with the Prader-Willi-like phenotype and with the selected years of publication (2000-2013). If two or more articles reported the same chromosomal alterations for Prader-Willilike phenotype, the most recent was chosen. Twelve articles of 14 were case reports and 2 reported series of cases.

Key words: Prader-Willi-like phenotype; Prader-Willi syndrome; Obesity; Mental deficiency

\section{INTRODUCTION}

Prader-Willi syndrome (PWS) is a disorder characterized by neurogenetic, neurometabolic and neurobehavioral alterations. Its level of incidence is between 1:10,000 and 1:20,000 live births, with 350,000 to 400,000 people affected in the world (Bittel and Buttler, 2005). Its main clinical features are hypotonia, hyperphagia, obsesity, hypogonadism, short stature, small hands and feet, mental disabilities, and behavioral problems (Cassidy, 1997; Cassidy and Driscoll, 2008). Most of these phenotypic manifestations, such as short stature, hypogonadism and hyperphagia are related to a dysfunction in the hypothalamus (Goldstone, 2004; Wattendorf and Muenke, 2005). It is a human multisystemic complex genetic disorder due to the lack of expression of paternal genes on chromosome 15q11-q13. Therefore, there are three main classes of chromosomal abnormalities that lead to PWS: deletion on 15q11-q13, maternal uniparental disomy (mUPD) of chromosome 15, or a defect in the imprinting center on 15q11-q13, although gene mutation $(<0.1 \%)$ and balanced translocation $(0.1 \%)$ can also be found (Figure 1). It is important to mention that deficiency of paternal 15q11-q13 is an important cause of syndromic obesity in humans.

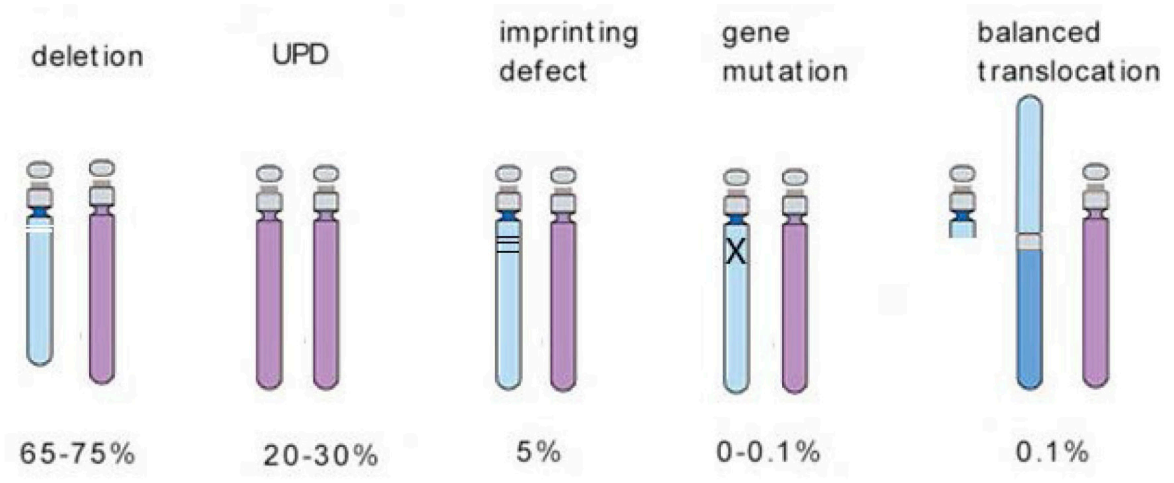

Figure 1. Ideograms showing possible causes of chromosomal abnormalities in Prader-Willi syndrome. In light purple, maternal chromosome 15 ; in blue, the paternal chromosome 15 . UPD $=$ uniparental disomy. 
It is not easy to see a deletion on 15q11-q13 using an ordinary light microscope. This may be due to the difficulty in visualizing a 3-5 $\mathrm{Mb}$ deletion with a light microscope and differences in condensation of band 15q12 (Varela et al., 2002). Therefore, G-banding is not sufficient to diagnose PWS (Gillessen-Kaesbach et al., 1995). To get an accurate diagnosis it is necessary to apply molecular techniques, such as fluorescence in situ hybridization (FISH) to confirm whether or not a chromosomal deletion is present (Figure 2) (Borelina et al., 2004), as well as the PCR-based methylation test (PBMT) for amplification of 15q11-q13, which is the gold standard technique for detection of the three main etiological genetic classes of PWS.

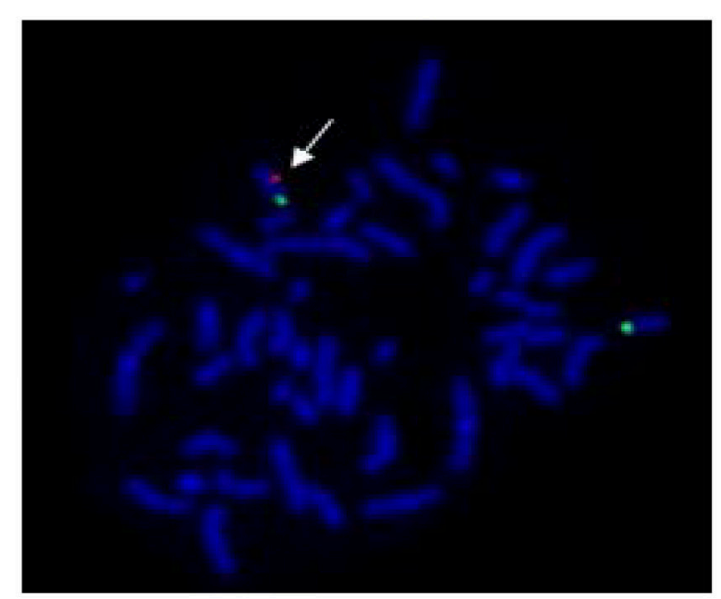

Figure 2. Metaphasic chromosomes subjected to hybridization with fluorescent probes (FISH) for $S N R P N$ and 15 qter. Green dot: 15 qter probe; red dot: $S N R P N$ probe. White arrow indicates the hybridization of the red probe SNRPN.

The challenge for the scientific community is not only to differentiate more clearly between PWS and the various PW-like phenotypes on a clinical level but also to provide conclusive genetic explanations for these phenotypes to provide accurate genetic counseling and treatment. Therefore, clinicians face a challenge in determining when to request the specific molecular test used to identify patients with classical PWS because the signs and symptoms of PWS are common to other syndromes (Cassidy and Driscoll, 2008). The absence of a correct diagnosis may worsen the prognosis of these individuals due to the endocrine-metabolic malfunctioning associated with the PW phenotype. Therefore, an accurate chromosomal investigation is necessary to differentiate classical PWS from the PW-like phenotype.

Our aim was to review the literature from 2000 to 2013, to shed light on Prader-Willilike phenotypes. This phenotype is caused by mutations other than those on chromosome 15 . This means that we included in this mini-review the patients who were clinically diagnosed as having the PW-like phenotype, since they had signs and symptoms compatible with classical PWS but were negative for alterations on 15q11-q13.

\section{MATERIAL AND METHODS}

We reviewed the literature between 2000 and 2013 for cases of PW-like phenotype 
caused by mutations other than those on chromosome 15. A search was carried out using the "National Center for Biotechnology Information" (www.pubmed.com) and "Scientific Electronic Library Online (www.scielo.br) databases and combinations of key words such as "Prader-Willi-like phenotype" and "Prader-Willi syndrome phenotype". Editorials, letters, reviews, and guidelines were excluded.

\section{RESULTS}

Our search found 643 articles about PWS, but only 14 of these matched with the Prader-Willi-like phenotype and with the selected years of publication (2000-2013). If two or more articles reported the same chromosomal alterations for PW-like phenotype, the most recent was chosen. Twelve articles of 14 were case reports (Lukusa and Fryns, 2000; De Molfetta et al., 2002; Florez et al., 2003; Stalker et al., 2003; Niyazov et al., 2007; Nowicki et al., 2007; Gabbett et al., 2008; Pramyothin et al., 2010; Tsuyusaki et al., 2010; Ben-AbdallahBouhjar et al., 2012; Doco-Fenzy et al., 2013; Izumi et al., 2013) and two reported series of cases: one of 78 cases (Hosoki et al., 2009) and another of 9 cases (D'Angelo et al., 2013) (Table 1). The patients included in this review were clinically diagnosed as bearing the PWlike phenotype because they were negative for alterations on 15q11-q13.

The authors cited in our review evaluated a total of 117 PW-like patients. Of these 117 patients, 44 had their final genetic diagnosis established. Their most frequent symptoms were obesity (84\%), hyperphagia (72.7\%), mental disability (54.5\%), psychomotor delay (50\%), and hypotonia (43.18\%).

At first sight, the above characteristics led to initial clinical diagnoses of PWS. To confirm the diagnosis, a variety of genetic tests were performed, such as PBMT, FISH and karyotype analysis, as well as comparative genomic hybridization (CGH). After the application of these techniques, the authors were able to exclude classical PWS, i.e., the syndrome caused by deficiency on 15q11-q13. They investigated further and discovered other mutations that could be associated with the PW-like phenotype, such as: a molecular pattern compatible with Angelman's syndrome (De Molfetta et al., 2002); chromosome 14 maternal uniparental disomy (Hosoki et al., 2009); monosomy of 1p36 (Tsuyusaki et al., 2010); deletion of 6q (Izume et al., 2013), 2pter deletion (Doco-Fenzy et al., 2013); and 10q26 deletion (Lukusa and Fryns, 2000); paracentric inversion (X)(q26q28) (Florez et al., 2003); 12q subtelomere deletions (Niyazov et al., 2007); Xq27-qter disomy; deletion 3p26.3 (Ben-Abdallah-Bouhjar et al., 2012); fragile X (Nowicki et al., 2007); and fragile X with 47,XYY (Stalker et al., 2003); deletion in 6q (Izumi et al., 2013); and Klinefelter syndrome karyotype, which showed a duplication of X(q21.1-q21.31) (Pramyothin et al., 2010) (Table 1). Recently, D'Angelo et al. (2013) reported different copy number imbalances of chromosomes 2, 3, 6, 10, 12, 14, and X, in nine patients showing the PW-like phenotype.

Maternal uniparental disomy of chromosome 14 was tested in 78 of 117 patients, and four disomies were encountered in this sample [three full upd(14)mat and 1 mosaicism], including one patient who had an epimutation in 14q32.2. The clinical features of the patients with epimutation or with mosaic upd(14)mat were not distinct from those of the patients with full upd(14)mat (Hosoki et al., 2009). Thirteen patients were positive for fragile X (Nowicki et al., 2007); 2/117 had a deletion in 1p36 (Tsuyusaki et al., 2010). 

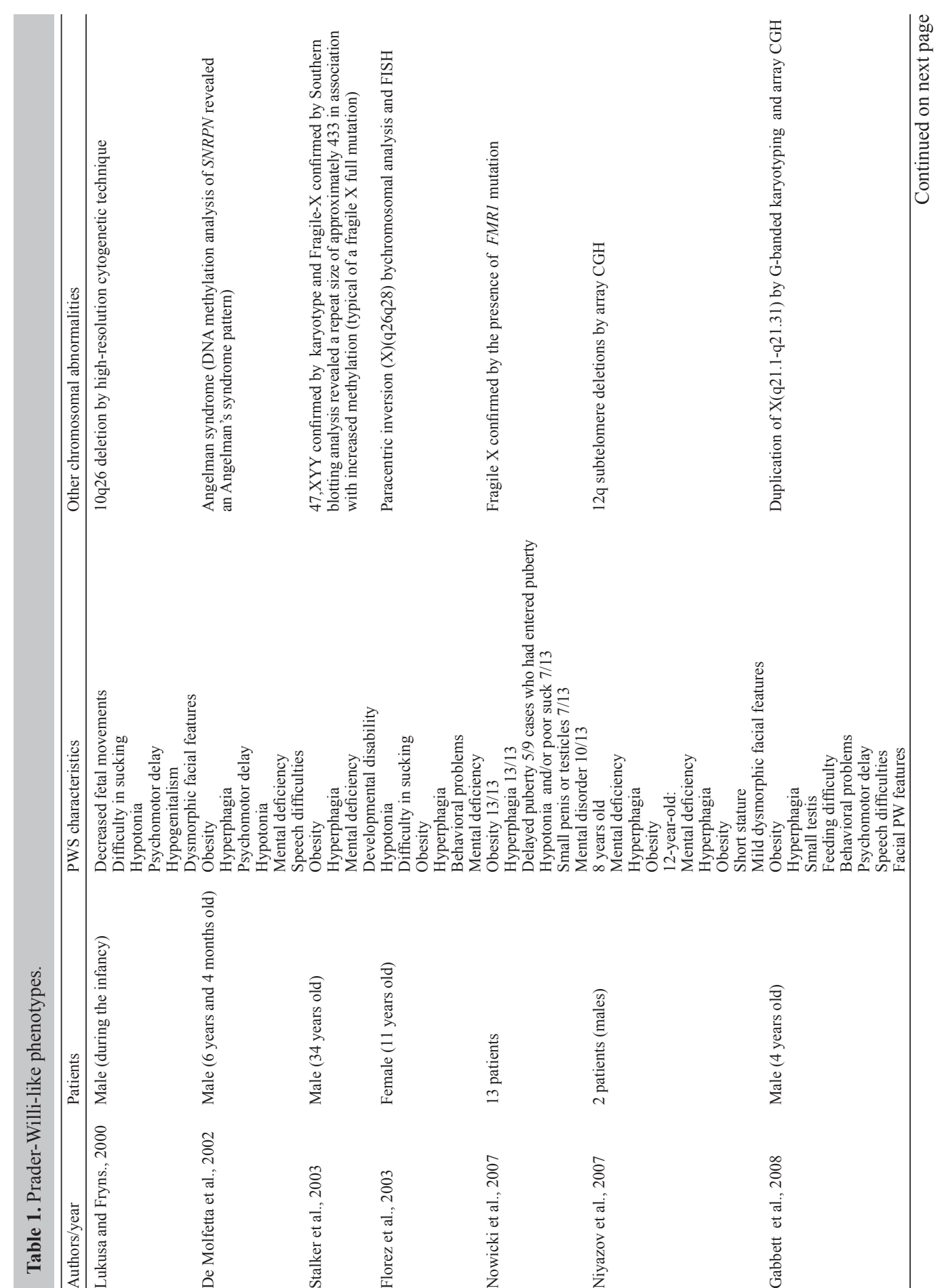

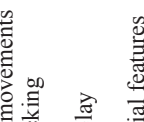
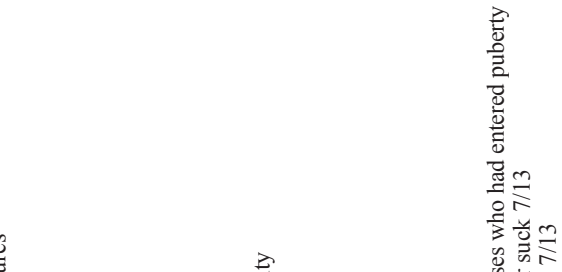

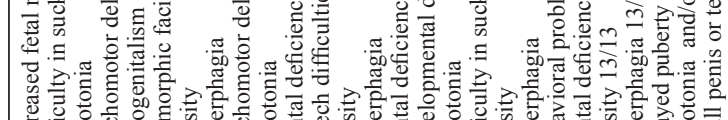

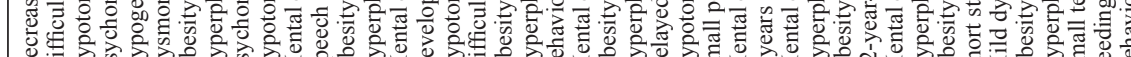

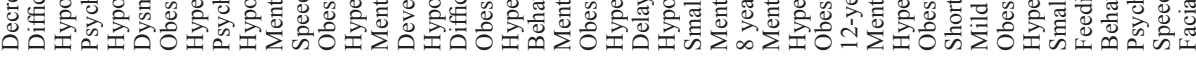

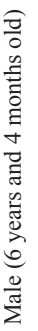

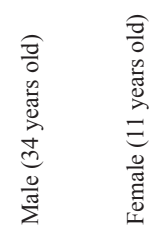

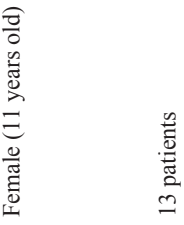

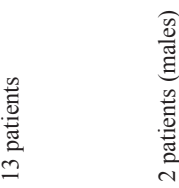

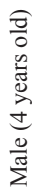

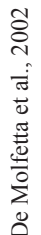

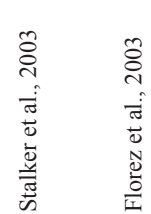

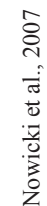

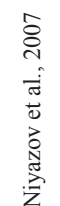

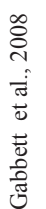

Genetics and Molecular Research 13 (1): 2290-2298 (2014) 
Prader-Willi-like phenotypes
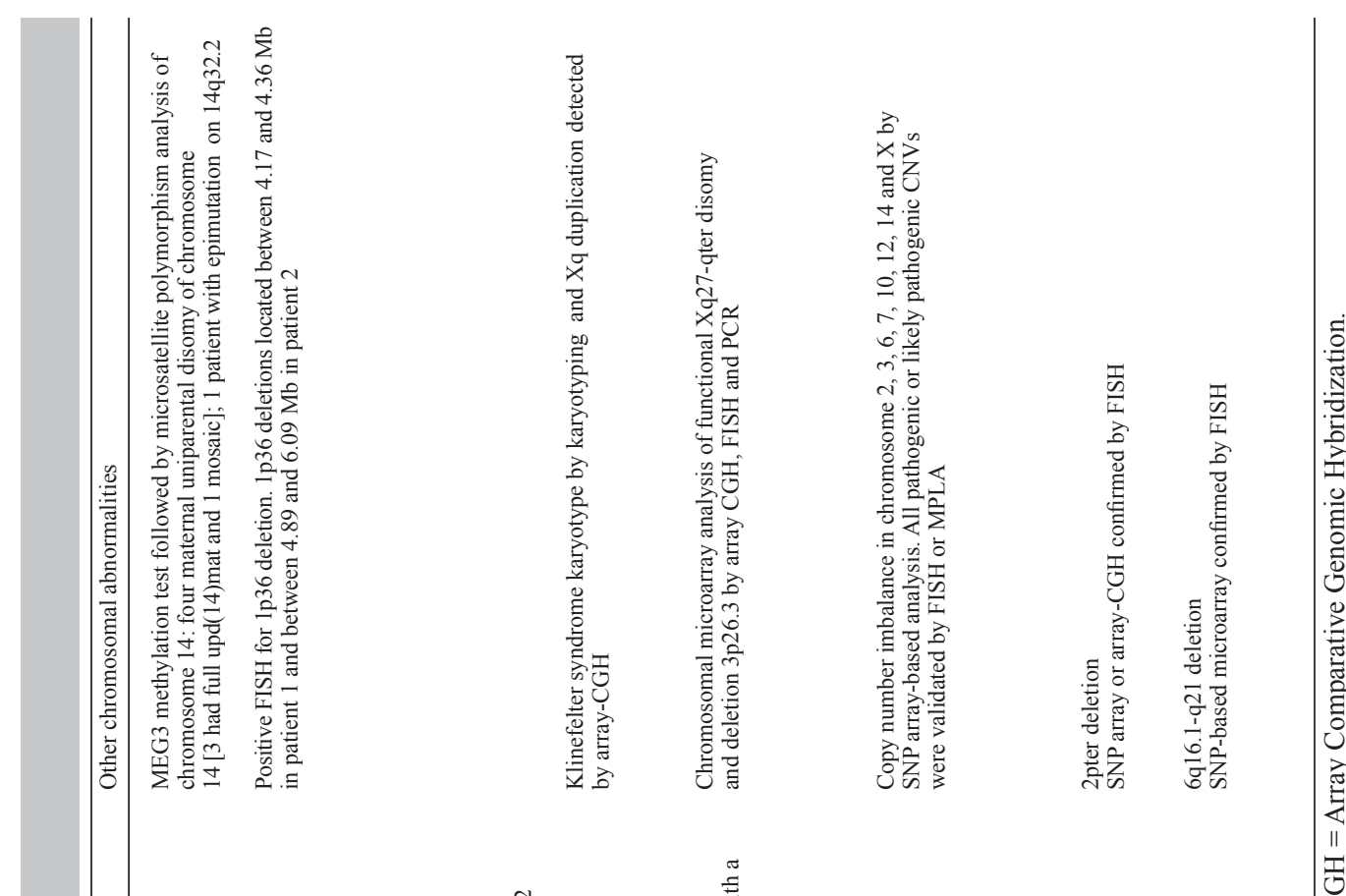

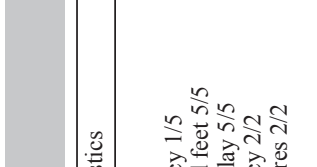

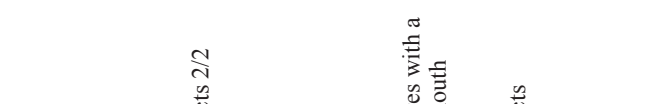

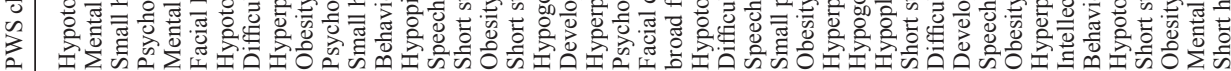

(

昰

$\frac{1}{2} \frac{0}{0}$

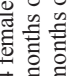

t是

0
0

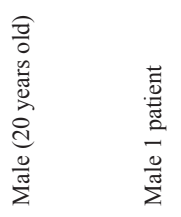

矛

离

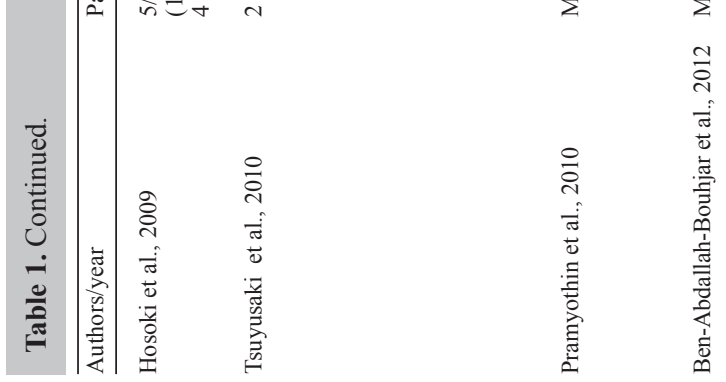

Genetics and Molecular Research 13 (1): 2290-2298 (2014)

CFUNPEC-RP www.funpecrp.com.br 


\section{DISCUSSION}

PWS has two distinct phases. The first is characterized by different degrees of hypotonia during the neonatal period and early childhood. Hypotonia is not progressive and begins to improve between 8 and 11 months of age, on average. When hypotonia improves, the child becomes more alert but hyperphagia starts. The onset of the second phase is marked by obesity, which usually occurs as a consequence of hyperphagia around 2 years of age. In this phase, children demonstrate small facial anomalies such as almond eyes, strabismus and thin upper lip. The patient also shows neuropsychomotor delay, short stature, small hands and feet, hypopigmentation of skin, hair and retina, learning problems, and pubertal delay (Butler, 1990; Holm et al., 1993; Cassidy, 1997; Fridman et al., 2000). Therefore, it is difficult to clinically diagnose PWS because some symptoms change with age and some characteristics are common to other pathologies. It is worth mentioning that significant obesity usually begins after hyperphagia has begun between the ages of 1 and 6 years (Cassidy, 1997), which makes early diagnosis difficult. Thus, PWS is often not clinically recognized in infants and, on the other hand, is wrongly suspected in obese and mentally deficient patients (Gillessen-Kaesbach et al., 1995).

We aimed with this article to review the different PW-like phenotypes and their respective chromosomal abnormalities (Table 1). PWS was excluded in the majority of the PWlike patients presented in this review by means of PBMT specific for this chromosomal region. This method can detect the three most frequent etiological classes of PWS such as deletion, mUPD or defective genomic imprinting on paternal 15q11-q13 (Cassidy and Driscoll, 2008).

It is important to emphasize that signs and symptoms of PWS could also be found in patients who show other types of chromosomal abnormalities such as a duplication of Xq (Gabbett et al., 2008), 1p36 monosomy (Tsuyusaki et al., 2010), a 6q deletion (Izumi et al., 2013), or fragile X (Stalker et al., 2003; Nowicki et al., 2007). Therefore, these patients exhibited a PW-like phenotype. Thus, it is necessary to routinely exclude fragile X syndrome from the diagnosis when the patients present mental deficiency and/or psychomotor delay.

Our literature review also showed up other chromosomal abnormalities that were less commonly cited as associated with the PW-like phenotype, such as the 10q26 deletion (Lukusa and Fryns, 2000), 12q subtelomere deletions (Niyazov et al., 2007), chromosomal abnormality associated with Angelman's syndrome (De Molfetta et al., 2002), 2pter deletion (Doco-Fenzy et al., 2013), and other X chromosome abnormalities (Florez et al., 2003; BenAbdallah-Bouhjar et al., 2012). The resultant phenotype of the duplications of the long arm of the $\mathrm{X}$ chromosome is more severe in males because of modulation by skewed $\mathrm{X}$-inactivation in females. Therefore, clinical manifestations widely vary depending on the gender of the patient and on the gene content of the duplicated segment. Nowadays, the growing number of Xq duplications can be described with the widespread use of array CGH techniques. Microarray analysis in clinical practice has facilitated the identification of novel obesity-associated syndromes, usually associated with learning disability and/or developmental delay.

A causal relationship has been recognized in both monogenic (e.g., $B D N F, T R K B$, and SIM1 deficiencies) and syndromic forms of obesity (e.g., PWS). On the other hand, genotypephenotype correlation is critical to determine the effects of novel copy number variants in patients associated with obesity and learning disability (D'Angelo et al., 2013).

It is important to mention that the majority of suspected patients who had a negative result for the specific PBMT for PWS had not shown the main features of PWS, such as neonatal hypotonia, feeding problems, facial features, or hypogonadism. Therefore, none of the ar- 
ticles included in this review presented patients bearing all of the PWS clinical characteristics.

Gilhuis et al. (2000) described a case and reviewed the literature about obese patients with alterations in the long arm of chromosome 6 . They found that these patients had in common obesity, hypotonia and delayed development similar to PWS. One explanation for this phenotype could be the presence of gene SIM1 haplodeficiency causing a defect in the leptinmelanocortin-oxytocin pathway (Holder et al., 2000; Tolson et al., 2010).

A comparative study investigating the prevalence and severity of obsessive-compulsive symptoms (OCS) in PWS and Prader-Willi-like syndrome showed that PWS patients suffered a higher incidence of OCS and more severe symptoms than their PW-like counterparts (State et al., 1999). Fridman et al. (2000) emphasized that the PBMT specific for PWS should be requested for all neonates and infants showing hypotonia, the most frequent clinical feature found in the patients considered in this review. However, other phenotypic characteristics must also be present to make an early diagnosis of PWS, such as small hands and feet, skin hypopigmentation, almond eyes, a narrow forehead and/or feeding difficulties. It is worth mentioning that an early and precise diagnosis of PWS is essential for avoiding obesity and related co-morbidities. It also allows for the provision of adequate genetic counseling for the patients and their families.

\section{CONCLUSION}

The challenge for the scientific community is not only to differentiate more clearly between PWS and the various PW-like phenotypes on a clinical level, but also to come up with conclusive genetic explanations for these phenotypes, to provide accurate genetic counseling. Although, the PCR-based methylation test is the gold standard technique for detecting PWS, cytogenetic analysis should also be attempted, not only to look for a 15q11-q13 deletion, but because other chromosomal abnormalities can be identified by the same technique. There is no doubt that new clinically recognizable syndromes will soon be described using the CGH technique, allowing targeted diagnosis.

\section{ACKNOWLEDGMENTS}

Research supported by Coordenação de Aperfeiçoamento de Pessoal do Ensino Superior (CAPES), Universidade Federal do Estado do Rio de Janeiro (UNIRIO) and Financiadora de Estudos e Projetos (FINEP).

\section{REFERENCES}

Ben-Abdallah-Bouhjar I, Hannachi H, Labalme A, Gmidene A, et al. (2012). Chromosomal microarray analysis of functional Xq27-qter disomy and deletion 3p26.3 in a boy with Prader-Willi like features and hypotonia. Eur. $J$. Med. Genet. 55: 461-465.

Bittel DC and Butler MG (2005). Prader-Willi syndrome: clinical genetics, cytogenetics and molecular biology. Expert Rev. Mol. Med. 7: 1-20.

Borelina D, Engel N, Esperante S, Ferreiro V, et al. (2004). Combined cytogenetic and molecular analyses for the diagnosis of Prader-Willi/Angelman syndromes. J. Biochem. Mol. Biol. 37: 522-526.

Butler MG (1990). Prader-Willi syndrome: current understanding of cause and diagnosis. Am. J. Med. Genet. 35: 319-332.

Cassidy SB (1997). Prader-Willi syndrome. J. Med. Genet. 34: 917-923.

Cassidy SB and Driscoll DJ (2008). Prader-Willi syndrome. Eur. J. Hum. Genet. 17: 3-13. 
D'Angelo CS, Kohl I, Varela MC, de Castro CI, et al. (2013). Obesity with associated developmental delay and/or learning disability in patients exhibiting additional features: report of novel pathogenic copy number variants. Am. J. Med. Genet. A 161A: 479-486.

De Molfetta GA, Felix TM, Riegel M, Ferraz VE, et al. (2002). A further case of a Prader-Willi syndrome phenotype in a patient with Angelman syndrome molecular defect. Arq. Neuropsiquiatr. 60: 1011-1014.

Doco-Fenzy M, Leroy C, Schneider A, Petit F, et al. (2013). Early-onset obesity and paternal 2pter deletion encompassing the ACP1, TMEM18, and MYT1L genes. Eur. J. Hum. Genet. Doi: 10.1038/ejhg.2013.189.

Florez L, Anderson M and Lacassie Y (2003). De novo paracentric inversion (X)(q26q28) with features mimicking PraderWilli syndrome. Am. J. Med. Genet. A 121A: 60-64.

Fridman C, Kok F and Koiffmann CP (2000). Síndrome de Prader-Willi em lactentes hipotônicos. J. Pediatr. 76: 246-250.

Gabbett MT, Peters GB, Carmichael JM, Darmanian AP, et al. (2008). Prader-Willi syndrome phenocopy due to duplication of Xq21.1-q21.31, with array CGH of the critical region. Clin. Genet. 73: 353-359.

Gilhuis HJ, van Ravenswaaij CM, Hamel BJ and Gabreels FJ (2000). Interstitial 6q deletion with a Prader-Willi-like phenotype: a new case and review of the literature. Eur. J. Paediatr. Neurol. 4: 39-43.

Gillessen-Kaesbach G, Gross S, Kaya-Westerloh S, Passarge E, et al. (1995). DNA methylation based testing of 450 patients suspected of having Prader-Willi syndrome. J. Med. Genet. 32: 88-92.

Goldstone AP (2004). Prader-Willi syndrome: advances in genetics, pathophysiology and treatment. Trends Endocrinol. Metab. 15: 12-20.

Holder JL Jr, Butte NF and Zinn AR (2000). Profound obesity associated with a balanced translocation that disrupts the SIM1 gene. Hum. Mol. Genet. 9: 101-108.

Holm VA, Cassidy SB, Butler MG, Hanchett JM, et al. (1993). Prader-Willi syndrome: consensus diagnostic criteria. Pediatrics 91: 398-402.

Hosoki K, Kagami M, Tanaka T, Kubota M, et al. (2009). Maternal uniparental disomy 14 syndrome demonstrates PraderWilli syndrome-like phenotype. J. Pediatr. 155: 900-903.

Izumi K, Housam R, Kapadia C, Stallings VA, et al. (2013). Endocrine phenotype of 6q16.1-q21 deletion involving SIM1 and Prader-Willi syndrome-like features. Am. J. Med. Genet. A 161A: 3137-3143.

Lukusa T and Fryns JP (2000). Pure distal monosomy 10q26 in a patient displaying clinical features of Prader-Willi syndrome during infancy and distinct behavioural phenotype in adolescence. Genet. Couns. 11: 119-126.

Niyazov DM, Nawaz Z, Justice AN, Toriello HV, et al. (2007). Genotype/phenotype correlations in two patients with 12q subtelomere deletions. Am. J. Med. Genet. A 143A: 2700-2705.

Nowicki ST, Tassone F, Ono MY, Ferranti J, et al. (2007). The Prader-Willi phenotype of fragile X syndrome. J. Dev. Behav. Pediatr. 28: 133-138.

Pramyothin P, Pithukpakorn M and Arakaki RF (2010). A 47,XXY patient and Xq21.31 duplication with features of Prader-Willi syndrome: results of array-based comparative genomic hybridization. Endocrine 37: 379-382.

Stalker HJ, Keller KL, Gray BA and Zori RT (2003). Concurrence of fragile X syndrome and 47,XYY in an individual with a Prader-Willi-like phenotype. Am. J. Med. Genet. A 116A: 176-178.

State MW, Dykens EM, Rosner B, Martin A, et al. (1999). Obsessive-compulsive symptoms in Prader-Willi and "PraderWilli-Like" patients. J. Am. Acad. Child Adolesc. Psychiatry 38: 329-334.

Tolson KP, Gemelli T, Gautron L, Elmquist JK, et al. (2010). Postnatal Sim1 deficiency causes hyperphagic obesity and reduced Mc4r and oxytocin expression. J. Neurosci. 30: 3803-3812.

Tsuyusaki Y, Yoshihashi H, Furuya N, Adachi M, et al. (2010). 1p36 deletion syndrome associated with Prader-Willi-like phenotype. Pediatr. Int. 52: 547-550.

Varela MC, Fridman C and Koiffmann CP (2002). Diagnosis of patients with Prader-Willi and Angelman Syndromes: the importance of an overall investigation. Genet. Mol. Biol. 25: 7-12.

Wattendorf DJ and Muenke M (2005). Prader-Willi syndrome. Am. Fam. Physician 72: 827-830. 